\title{
Treatment of carrageenan induced arthritis by the platelet activating factor antagonist BN 50730
}

\author{
P Hilliquin, J Natour, J Aissa, P Guinot, S Laoussadi, J Benveniste, C J Menkes, B Arnoux
}

\begin{abstract}
Objective-To evaluate the role of platelet activating factor (PAF) in the early stage of arthritis.

Methods-Arthritis was induced in rabbits by weekly intra-articular injections of carrageenan. A PAF receptor antagonist, BN 50730, was used as a preventive or curative agent.

Results-BN 50730 was able partially to prevent the development of arthritis, and was also active on established arthritis. The joint arthritis scores of BN treated animals were significantly lower than those of the non-treated animals. The blood concentrations of PAF, PAF bound to lipoproteins (lipo-PAF), and its precursor, lyso-PAF, were not correlated with clinical variations.

Conclusions-The present data demonstrate a therapeutic action of a PAF antagonist in experimental arthritis and suggest a critical role for PAF during the early stage of arthritis.
\end{abstract}

(Ann Rheum Dis 1995; 54: 140-143)

Platelet activating factor (PAF) is a phospholipid mediator released by numerous inflammatory cells. ${ }^{1-5}$ It is a potent activator of neutrophils and monocytes. In human pathology, the effects of PAF have been studied mainly on pulmonary and cardiovascular systems, ${ }^{6}$ but its involvement in the amplification cycle of inflammatory reactions and its relationship with cytokines suggest it may be implicated in the physiopathology of arthritis. We recently reported the presence of PAF in blood and synovial fluid from patients with arthropathies, with increased concentrations in active rheumatoid arthritis. ${ }^{7} \mathrm{~A}$ PAF-degrading enzyme, acetylhydrolase, was also detected in RA in serum and synovial fluid. ${ }^{8}$

The present study was undertaken to evaluate the effects of blocking PAF in an experimental rabbit model of arthritis. Carrageenan, a mucopolysaccharide agent with proinflammatory properties, was used as the arthritogenic compound. The specific PAF receptor antagonist, BN $50730,{ }^{9}$ was used as a preventive or curative agent.

\section{Materials and methods}

INDUCTION OF ARTHRITIS

Arthritis was induced in New Zealand White rabbits (weights 3-4 kg) by five weekly intraarticular injections (days $0,7,14,21$, and 28) in the left knee of $10 \mathrm{mg}$ carrageenan lambda $1 \%$ (Sigma) diluted in $1 \mathrm{ml}$ saline. The right knee received no injection and served as a control. The PAF antagonist BN 50730 was administered intraperitoneally (ip) $(5 \mathrm{mg} / \mathrm{kg} /$ day).

Five groups of five animals were studiedgroup 1: intra-articular (ia) carrageenan without treatments; group 2: ia carrageenanBN 50730 ip from day 0 to day 60 ; group 3 : ia carrageenan-ip injections of $\mathrm{BN}$ solvent from day 0 to day 60 ; group 4 : ia carrageenanip $\mathrm{BN}$ treatment from day 28 to day 60 ; group 5: ia saline serum $1 \mathrm{ml}-\mathrm{ip} \mathrm{BN}$ treatment from day 0 to day 60 .

EVALUATION OF ARTHRITIS

Evaluation was performed at day 0, before each ia injection at days $7,14,21,28$, and at days 35,45 , and 60 . Arthritis was evaluated blindly by measuring the joint diameter at the femoro-tibial level, and by an arthritis score: $0=$ normal joint; $1=$ mild arthritis; $2=$ moderate arthritis; $3=$ severe arthritis. The scoring system was defined according to the importance of the tumefaction (synovitis, synovial fluid, or both) and local heat. Venous blood was collected for measurement of PAF and derivatives.

At day 60, all rabbits were sacrified (intravenous pentobarbitone), and both knees were dissected. Histological analysis was performed blind by two observers.

\section{DETECTION OF PAF AND DERIVATIVES}

Blood was collected on $100 \%$ ethanol. PAF and lipo-PAF were separated by high pressure liquid chromatography and assessed by their aggregating activity on washed rabbit platelets as described previously. ${ }^{10}$ PAF was characterised by its sensitivity to phospholipase $\mathrm{A}_{2}$ and the lack of effect of lipase A1 from Rhizopus arrhizus. ${ }^{11}$ Results are expressed as PAF equivalents ( $\mathrm{ng} / \mathrm{ml})$. Lyso-PAF was treated by chemical acetylation to generate PAF and assessed by platelet aggregation. ${ }^{12}$

\section{STATISTICAL ANALYSIS}

Clinical and biological parameters were analysed by multiple factors analysis of variance according to group and day. A multiple range test was performed when appropriate to determine differences between groups and days.

A first set of analyses was performed for the period 0-60 days. Two other analyses were performed with the same tests on clinical 
parameters: one on the period 0-21 days and the second for the period $28-60$ days.

\section{Results}

DEVELOPMENT OF ARTHRITIS

The animals from group 5, which received saline injections, did not develop arthritis during the study period.

Repeated ia injections of carrageenan induced arthritis in all rabbits, detectable at the end of the first week (fig 1). At day 7, the left knee arthritis score was greater for animals not treated with BN (groups 1, 3, and 4) (analysis of variance and multiple range test, $p<0.05$ ). In these groups, the maximal arthritis score was observed between two and three weeks. During the first four weeks, the non-treated rabbits exhibited a similar arthritis course. The BN solvent did not seem to have any particular effect, as rabbits from group 3 had arthritis scores quite similar to those of rabbits from group 1. For the non-treated animals (groups 1 and 3 ), the arthritis retained approximately the same intensity until the completion of the study.

During the first period (day 1 to day 28), BN treated rabbits (group 2) had lower arthritis scores than the non-treated animals (groups 1 , 3 , and 4) (analysis of variance, $\mathrm{p}<0.05$ ).

At day 28, the arthritis score was statistically identical in the three groups of animals which had not been previously treated with the PAF antagonist. However, when group 4 animals were then treated with PAF antagonist, the arthritis intensity score decreased dramatically to become identical to that of rabbits receiving the drug since day 0 .

A significant statistical difference (analysis of variance, $p<0.05$ ) was observed between the

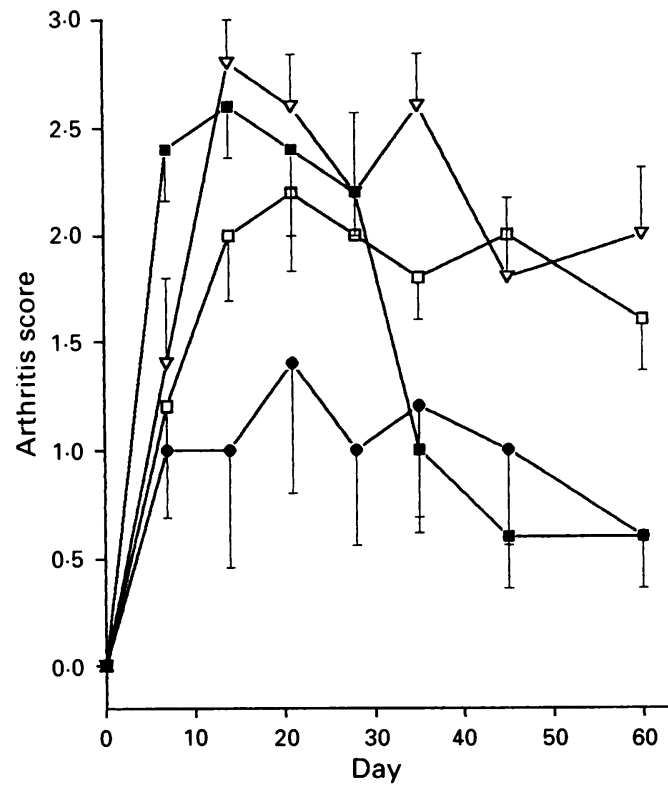

Figure 1 Clinical evaluation of arthritis by a score from 0 to 3 (mean, SEM) in rabbits. Group $1(\nabla)=i a$ carrageenan without any treatment; group $2(0)=i a$ carrageenan and BN 50730 ip from day 0 to day 60; group $3(\square)=i a$ carrageenan and ip injections of $B N$ solvent from day 0 to day 60 ; group $4(\square)=i a$ carrageenan and ip BN treatment from day 28 to day 60 . Arthritis was never detected in group 5, which is not represented on this figure. five groups for the arthritis score during the first period of analysis. The multiple range test identified three homogeneous groups: groups 1,3 , and 4 were statistically similar; group 2 exhibited a milder arthritis; group 5 (without arthritis) was different from the two other groups.

During the second period of analysis (28-60 days), a statistical difference was still present between groups, but the group distribution changed: groups 1 and 3 were identical, group 4 became similar to group 2 , and both were still different from group 5 . The same statistical outcome was obtained from the two indices of arthritis - left joint diameter or joint diameter difference between left and right knee (fig 2): there was a high correlation between arthritis score and left joint diameter $(r=0.90)$. The right knee (control, not injected) remained normal in all rabbits during the period of observation.

\section{BIOLOGICAL PARAMETERS}

An increase in circulating PAF was observed at day 7 in all groups. The greatest value was detected in group 2, without significant differences between groups. After the first two weeks, PAF was undetectable in most animals (fig 3).

Blood lipo-PAF and lyso-PAF exhibited cyclic variations in the five groups, without apparent modification related to the treatment. No statistically significant difference could be demonstrated between groups.

No correlation could be established between clinical and biological variables.

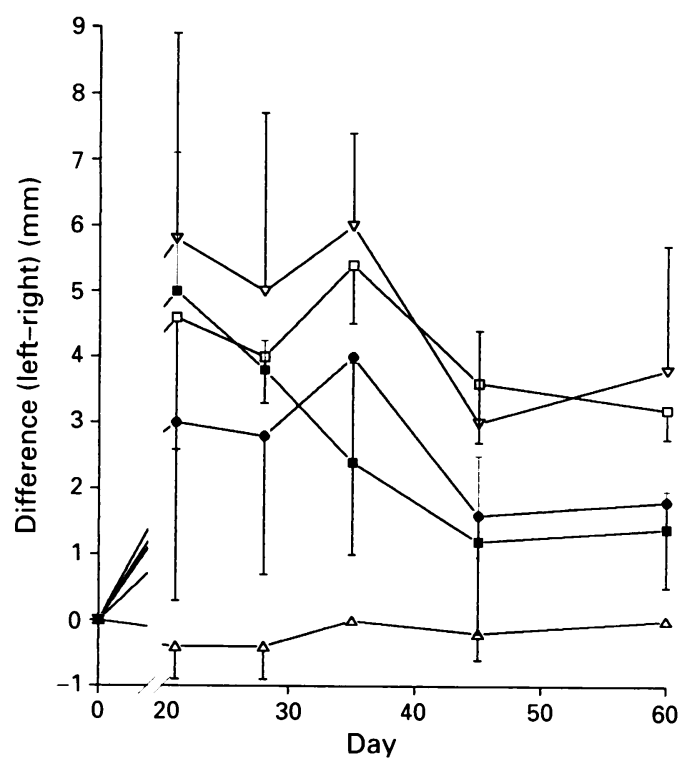

Figure 2 Clinical evaluation of arthritis by measurement of the knee joint at the femoro-tibial level. Results are expressed as the difference between the left and the right knee in five groups of rabbits. Group $1(\nabla)=i a$ carrageenan without any treatment; group $2(\mathrm{O})=i a$ carrageenan and $B N 50730$ ip from day 0 to day 60; group $3(\square)=i$ carrageenan and ip injections of $B N$ solvent from day 0 to day $60 ;$ group $4(\square)=i a$ carrageenan and ip BN treatment from day 28 to day 60 ; group $5(\triangle)=i$ a saline serum $1 \mathrm{ml}$ and ip $B N$ treatment from day 0 to day 60 . The measurements are represented from day 21, when arthritis achieved maximal intensity, with the greatest difference between the two knees in all groups, until day 60. 


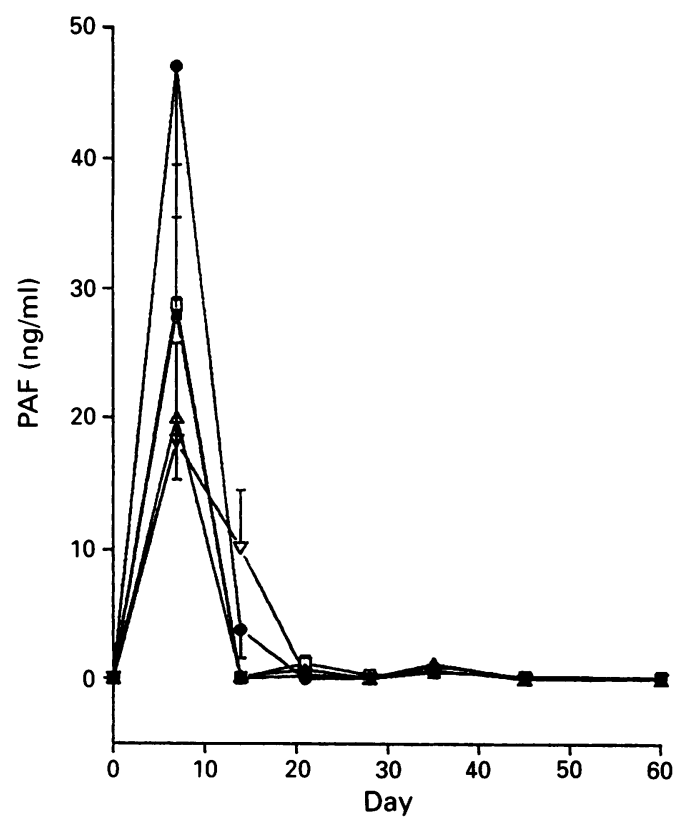

Figure 3 Mean (SEM) blood concentrations of PAF measured by washed rabbit platelet aggregation after HPLC separation in five groups of rabbits. Group $1(\nabla)=i a$ carrageenan without any treatment; group $2(0)=i a$ carrageenan and BN 50730 ip from day 0 to day 60 group $3(\square)=i$ carrageenan and ip injections of $B N$ solvent from day 0 to day $60 ;$ group $4(\square)=i a$ carrageenan and ip $B N$ treatment from day 28 to day 60 ; group $5(\triangle)=i$ a saline serum $1 \mathrm{ml}$ and ip $B N$ treatment from day 0 to day 60.

\section{HISTOLOGICAL ANALYSIS}

All right knees were normal. A synovitis was present in the left knee of all rabbits except those from group 5, and two rabbits from group 4. The histological appearance was that of a subacute synovitis, with lymphoplasmocyte infiltration and mild oedema. There was no difference in the synovitis of the animals from group 2 compared with groups 1 and 3.

\section{Discussion}

Very few data have been published concerning the implication of PAF in arthritis. The presence of high concentrations of PAF in synovial fluid from patients with arthritis suggests that PAF could act locally by recruiting proinflammatory cells to the joint cavity. Previous studies have shown that PAF can be produced by neutrophils present in the synovial fluid from patients with RA. ${ }^{13}$ More recent data also suggest that PAF can increase neutrophil mediated cartilage proteoglycan degradation and inhibit cartilage proteoglycan synthesis. ${ }^{14}$

In order to evaluate the implications for PAF in arthropathy, we tested the clinical effects of a PAF receptor antagonist in an experimental model of arthropathy induced by carrageenan, a proinflammatory mucopolysaccharide. We used the anti-PAF agent BN 50730 as preventive or curative agent.

Used prophylactically, BN 50730 was able partially to inhibit the development of arthritis, producing lower clinical scores than were obtained in the non-treated animals. Moreover, when administered after day 28 , it reversed the clinical arthritis scores, which became identical to those of rabbits treated prophylactically. The therapeutic effect was derived from the drug itself, as the $\mathrm{BN}$ solvent had no action.

The blood concentrations of PAF were not correlated with the variations in clinical presentation. The increase in PAF concentration at day 7 could be explained partly by the action of the PAF antagonist, competing with naturally occurring PAF for its binding sites; inhibition of PAF binding to its receptors can also block its cellular metabolism. ${ }^{15}$ Local production of PAF in the inflammatory site may also contribute to the increase in circulating PAF in the arthritic animals. Subsequent decreases in blood concentrations of PAF may have reflected activation of its degradation or its binding to blood lipoproteins.

Synovitis was demonstrated in all groups except the control group and the medication used was not able to inhibit the development of synovitis in all treated animals, despite clinical differences between groups.

Overall, we obtained one clear result: the impressive clinical effect of the PAF antagonist as both a protective and a curative agent. This gives further support to the putative role of PAF in the early stages of arthritis, but remains to be confirmed by other studies using PAF antagonists with different chemical structures. In contrast, PAF concentrations were not a good marker for the expression of the disease. Further studies are needed to evaluate the sources of PAF in the inflamed joint, its actions on synovial cells and chondrocytes, and its relationships with cytokines. The present data also suggest that PAF antagonists should be evaluated in animal models of arthritis which are more akin to the inflammatory arthropathies in man than is the carrageenan model.

This work was supported by a grant from Institut Henri Beaufour, Paris, France. We want to thank R Amsellem for he technical assistance.

1 Benveniste J, Tencé $M$, Varenne P, Bidault J, Boullet C, Polonsky J. Semi-synthèse et structure proposée du facteur activant les plaquettes (PAF): paf-acéther, un alkyl éther analogue de la lysophosphatidylcholine. C R Acad Sci Paris 1979; 289D: 1037-40.

2 Lotner G Z, Lynch J M, Betz S J, Henson P M. Human neutrophil-derived platelet-activating factor. $f$ Immunol 1980; 124: 676-84

3 Jouvin-Marche E, Ninio E, Beaurain G, Tence $M$ Niaudet P, Benveniste J. Biosynthesis of paf-acether (platelet-activating factor). VII. Precursors of paf-acether (platelet-activating factor). VII. Precursors of paf-acether and acetyl-transferase activity

4 Camussi G, Aglietta $M$, Malavasi $F$, et al. The release of platelet-activating factor from human endothelial cells in culture. F Immunol 1983; 131: 2387-403.

5 Chap H, Mauco G, Simon M F, Benveniste J, DousteBlazy L. Biosynthetic labelling of platelet-activating factor from radioactive acetate by stimulated platelets. Nature 1981; 289: 312-4

6 Arnoux B, Joseph M, Simoes M H, Capron A Benveniste J. Antigenic release of paf-acether and betaglucuronidase from alveolar macrophages of asthmatics. Bull Eur Physiopathol Respir 1987; 23: 119-24.

7 Hilliquin P, Menkes C J, Laoussadi S, Benveniste J, Arnoux B. Presence of paf-acether in rheumatic diseases. Ann Rheum Dis 1992; 51: 29-31.

8 Dulioust A, Hilliquin P, Menkes C J, Benveniste J, Arnoux B. Paf-acether acetylhydrolase activity is increased in patients with rheumatic diseases. Scand $\mathscr{f}$ Rheumatol 1992; 21: 161-4.

9 Dyson M C, Bellan J A, Minkes R K, et al. Influence of SK\&F 95587 and BN 50730 on bronchoconstrictor responses in the cat. $\mathcal{f}$ Pharmacol Exp Ther 1990; 255: 1320-7. 
10 Benveniste J, Henson P M, Cochrane C G. Leukocytedependent histamine release from rabbit platelets: the role
of IgE, basophils and a platelet-activating factor. $\mathcal{F} \operatorname{Exp}$ of IgE, basophils and a

11 Benveniste J, Le Couedic J P, Polonsky J, Tence M. Structural analysis of purified platelet activating factor by lipases. Nature 1977; 269: 170-1.

12 Polonsky J, Tence M, Varenne P, Das B C, Lunel J, Benveniste J. Release of 1-O-alkyl-glyceryl-3-phosphorylcholine, O-deacetyl platelet-activating factor, from leukocytes: chemical ionisation mass spectrometry of phospholipids. Proc Natl Acad Sci USA 1980; 77: 7019-23.
13 Gregoir C, Dulioust A, Hilliquin P, Laoussadi S, Arnoux A, Menkès C J. Production de paf-acéther par les neutrophiles du liquide synovial de patients ayant une polyarthrite rhumatoïde. Rev Rhum 1991; 58: 667.

14 Kowando I C, Bates E J, Ferrante A. Platelet-activating factor inhibits proteoglycan synthesis and enhances neutrophil-mediated proteoglycan degradation in cartilage explants. Arthritis Rheum 1992; 35: 918-25.

15 Lachachi H, Plantavid M, Simon M F, Chap H, Braquet $P$, Douste-Blazy L. Inhibition of transmembrane movement and metabolism of platelet-activating factor (PAF-acether) by a specific antagonist BN 52021 . (PAF-acether) by a specific antagonist BN
Biochem Biophys Res Commun 1985; 132: 460-6. 